AperTO - Archivio Istituzionale Open Access dell'Università di Torino

\title{
METABOREFLEX ACTIVITY IN HEART FAILURE WITH REDUCED AND PRESERVED EJECTION FRACTION
}

This is a pre print version of the following article:

Original Citation:

Availability:

This version is available http://hdl.handle.net/2318/1737778

since 2020-04-30T00:03:35Z

Published version:

DOI:10.1096/fasebj.2020.34.s1.03246

Terms of use:

Open Access

Anyone can freely access the full text of works made available as "Open Access". Works made available under a Creative Commons license can be used according to the terms and conditions of said license. Use of all other works requires consent of the right holder (author or publisher) if not exempted from copyright protection by the applicable law. 


\title{
Pre-print_Submitted to
}

\author{
The Faseb Journal
}

\section{METABOREFLEX ACTIVITY IN HEART FAILURE WITH REDUCED AND PRESERVED EJECTION FRACTION}

\author{
Silvana Roberto Pasquale Pagliaro Raffaele Milia Gabriele Mulliri Antonio Crisafulli
}

\begin{abstract}
Introduction

Chronic heart failure (CHF) is a syndrome characterized by reduced cardiac output (CO) unable to meet metabolic demands of active tissues. The reduced (HFrEF) or preserved ejection fraction (HFpEF) identifies the systolic and diastolic HF respectively. Evidence has demonstrated that metabolic reflex coming from skeletal muscle evokes cardiovascular adjustments during exercise. Hemodynamic response to metaboreflex activation is defined as a rise in mean arterial blood pressure (MAP) achieved by an increase in systemic vascular resistance (SVR) and/or by an increase in CO. In healthy individuals, the metaboreflex-induced increase in blood pressure mainly depends on $\mathrm{CO}$ increment. The aim of the present study was to assess cardiovascular adjustments during metaboreflex activation in both kinds of CHF patients compared to a control group of healthy individuals (CTL).
\end{abstract}

\section{Methods}

Three groups of age-matched subjects were studied: 1) 12 males patients diagnosed with HFrEF; 2) 8 males patients with diagnosed HFpEF; 3) 12 healthy individuals (CTL). All participants underwent a general medical examination, an incremental exercise test on a cycle ergometer to test their physical capacity, and a metaboreflex activation protocol consisting in a post exercise muscle ischemia (PEMI) and in a control exercise recovery (CER) session. All hemodynamic parameters were measured non-invasively by impedance cardiography.

\section{Results}

Statistical analysis showed no differences in heart rate (HR) response among groups. Stroke volume (SV) and CO responses were not significantly different between the two groups of HF patients. SV and CO responses were significantly higher in the CTL than in both the HFrEF and the HFpEF group. SVR response was significantly higher in the HFrEF as compared to the CTL group, while no differences existed between the CTL and the HFpEF. MAP response to PEMI was not different among groups. Ventricular Filling Rate (VFR, a measure of diastolic function) response was significantly higher in CTL group in comparison with the HFrEF and the HFpEF groups. Ventricular Emptying Rate (VER, a measure of systolic function) response was significantly higher in CTL group as compared to the two groups of patients.

\section{Conclusion}

Notwithstanding the described reduced capacity to enhance CO, the MAP response was not different among groups. This indicates that the possibility to reach an effective MAP pressure was unaltered in both groups of patients with CHF. This was likely the consequence of an exaggerated vasoconstriction due to a sympathetic overdrive in both groups of CHF patients. These findings are in good agreement with previous research dealing with HFrEF and HFpEF, which demonstrated the blood pressure rise during the metaboreflex is mainly achieved by a vasoconstriction-mediated rather than a flow-mediated mechanism. Of note the SVR increase was more accentuated in the HFrEF than in the HFpEF group, and this phenomenon suggests that the cardiovascular impairment was more pronounced in the former than in the latter group of patients. 